\title{
LA NOCIÓN DE VERLEGENHEIT EN KANT
}

\author{
RAFAEL REYNA
}

Universidad de Málaga

\section{Introducción}

En primer lugar, hay que señalar una característica de todo filósofo, a saber: todo filósofo tiene un problema para el que toda su filosofía es una respuesta. Si observamos la historia de la filosofía esto parece evidente. Descartes busca un fundamento del saber tras la filosofía de Ockham, también se dice que toda la filosofía posterior a Hegel es con Hegel y contra Hegel. Por tanto, el caldo de cultivo en el que se gesta la filosofía del autor que se estudie plantea a éste la problemática. Así, la fillosofía de Kant no es sólo una mera ocurrencia sino la respuesta a un problema. Se suele citar una frase de Kant para resumir su filosofía: "Wenn aber gleich alle unsere Erkenntnis mit der Erfahrung anhebt, so entspringt sie darum doch nicht eben alle aus der Erfahrung". Sin embargo, si queremos tener un conocimiento más profundo de Kant y no sólo del Kant post-crítico, tendremos que buscar en el Kant pre-crítico aquello que le hizo llevar a cabo el célebre giro copernicano.

Para comenzar, podríamos decir que el lugar de la obra de Kant donde se resume ese problema al que se viene aludiendo no es otro que el comienzo del primer prólogo de la Crítica de la Razón Pura: "Die menshliche Vernunft hat das besondere Schicksal in einer Gattung ihrer Erkenntnisse: daß sie durch Fragen belästigt wird, die sie nicht abweisen kann, denn sie sind ihr durch die Natur der Vernunft selsbt aufgegeben, die sie aber auch nicht beanworten kann, denn sie übersteigen alles Vermögen der menschelichen Vernunft [...] In diese Verlegenheit gerät sie ohne ihre Schuld"2.

\footnotetext{
${ }^{1}$ KANT, Immanuel. Kritik der reinen Vernunft. Frankfurt, 1781. Pág. 45 ."Todo el conocimiento se alza con la experiencia, pero no por eso todo el brota de la experiencia"

2 Íbidem, Pág. 11. "La razón tiene el destino especial, en un tipo de sus conocimientos, de ser acosada por preguntas que no puede rechazar, pues ellas mismas son dadas por la naturaleza de la razón, pero las cuales no pueden ser respondidas, pues ellas superan todas las capacidades de la razón humana [...] En esa perplejidad cae la razón sin su culpa.
} 


\section{El dogmatismo pre-crítico de Kant}

Kant, como filósofo moderno que es, tiene a la ciencia en alta estima y, aunque no la considera el único modo de conocimiento, si la considera como el modo más alto de conocimiento. La controversia entre Leibniz y Newton tiene gran importancia en esta época y Kant toma parte de Newton, aunque hay textos en los que el propio Kant señala que no tomará parte de ninguno de los dos. Kant no considera tan importante la matemática como la física, es decir su aplicación. Así, Kant concibe que hay leges entis que determinan la realidad y a las que el conocimiento científico ha podido llegar y esto es la causa del éxito que posee la ciencia en aquella época. Kant, por tanto, posee una gran admiración por la física de Newton y esto conduce a una visión del mundo acorde con dicha admiración.

El éxito de la ciencia se debe, pues, a la aplicación de la matemática a la física. Por tanto, parece evidente el siguiente paso será aplicar la matemática a la filosofía. Sin embargo, Kant en el Prólogo al Ensayo para introducir el concepto de magnitudes negativas distingue dos maneras de aplicar la matemática. La primera manera tiende a imitarla, ejemplo de ello es Spinoza, la segunda manera es la que Kant propone y consiste en la aplicación efectiva de las proposiciones matemáticas a los objetos de la filosofía ${ }^{3}$.

Por otra parte, si queremos entender al Kant pre-crítico es especialmente importante la obra Investigación sobre la claridad de los principios de la teología natural y de la moral. En dicha obra Kant da tres aportaciones:

1) La matemática es sintética mientras que la filosofía es analítica. Así, la matemática construye sus propios objetos a la vez que las definiciones, es decir, en cuanto que hay definiciones hay objetos. En función de esto mismo no hay, pues, demostraciones de las definiciones sino que éstas son ya la base de la matemática. Por su parte, la filosofía es analítica y supone los objetos de sus definiciones y pretende llegar, a través de abstracciones a la esencia misma de las cosas. Para Kant éstas no son más que imposiciones. La matemática, por tanto, tienes menos posibilidad de error y más certeza subjetiva. Dicha certeza subjetiva viene dada por los medios que usan una y otra. La matemática usa signos visibles y sensibles mientras que la filosofía usa palabras. Por tanto, se puede ya decir la noción de certeza para Kant es la de comprobación intuitiva. La comprobación no será, pues, otra cosa que la unidad de objeto y signo.

2) Aparecen ya las dos reglas para determinar cómo es el método óptimo de aplicación de la matemática a la filosofía. Estas reglas son: 1) no partir de definiciones,

3 Cfr. IGNACIO FALGUERAS, Perplejidad y filosofia trascendental en Kant. Pamplona, Universidad de Navarra, 1999, pág. 27. 
sino de certezas en orden a emitir juicios sobre los objetos más allá de la definición y 2) destacar los juicios inmediatos a partir de los datos y anteponerlos como axiomas geométricos para ser la base a conclusiones ulteriores ${ }^{4}$. Lo central es operar con lo que ya se sabe. Aparece aquí el problema del dato, o sea un punto desde donde se pueda erigir la metafísica. En cualquier caso, este problema es recurrente en la filosofía moderna, la búsqueda de un fundamento de todo el conocimiento. Así, se puede ver que el Kant precrítico no es tan novedoso como el post-crítico.

3) Kant señala que la metafísica aun no se ha construido y que él espera que, con su método, se puedan obtener síntesis de conocimientos. Además dice que no le basta sólo con síntesis sueltas, sino con conjuntos de síntesis que le permitan hacer agrupaciones. "Pues bien, la esperanza de que utilizando el método antes descrito se puedan alcanzar en la filosofía, a partir del análisis de los datos experienciales, conocimientos sintéticos semejantes a los de las matemáticas (síntesis a priori), es lo que creo que debe entenderse por sueño dogmático"s. Así lo apunta Kant en la Crítica de la Razón Pura: "Daher hoft reine Vernunft, im traszendentalen Gebrauche sich eben so glücklich und gründlich erweitern zu können, als es ihr im mathematischen gelungen ist, wenn sie vornehmlich dieselbe Methode dort anwendet, die hier von so augenscheimlichen Nutzen gewesen ist" ${ }^{\text {. }}$

Este es el sueño (Schlummer) en que se encontraba Kant, o sea, una situación en la que el regiomontano tenía las esperanzas de llegar a una aplicación del método matemático a la metafísica y obtener así un éxito igual que el obtenido por la física. Pues bien, este sueño no deja de ser un sueño y es con la crítica de la razón a sí misma como ella podrá salir de dicho sueño y así lo dice Kant: "so ist es notwendig, noch gleichsam den letzen Anker einer phantasiereichen Hoffnung wegzunehmen, und zu zeigen, daß die Befolgung der mathematischen Methode in dieser Art Erkenntnis nocht den mindesten Vorteil schaffen könne" ${ }^{\text {" }}$ ¿A qué se debe esta transformación del Kant más ilusionante al Kant post-crítico? ¿Cómo se advierte de que no es posible una síntesis intelectual allende a la experiencia? Pues, lo que se propone es que la culpable de dicho cambio no es otra cosa que la Perplejidad (Verlegenheit). Ésta es, pues, la piedra de toque de todo el sueño dogmático pre-crítico y el punto de partida de la Crítica de la Razón Pura. La toma de conciencia de

4 FALGUERAS, Ignacio, "Perplejidad y filosofia trascendental en Kant". Pamplona, Universidad de Navarra, 1999, pág. 31.

5 Íbidem. Pág.33

${ }^{6}$ KANT, Immanuel. Kritik der reinen Vernunft II. Pág. 613."En su uso trascendental, la razón espera, pues, conseguir extenderse con la misma solidez con que lo ha hecho en las matemáticas, especialmente si usa en el primer caso el método que tan palpables ventajas ha demostrado en el segundo" trad. Pedro Ribas

${ }^{7}$ A 726, trad. P. Ribas, 582. "Por ello, es necesario desprenderse del último ancla de una esperanza fantástica , por así decirlo, y mostrar que la práctica del método matemático es incapaz de reportar el menos beneficio en este tipo de conocimiento" trad. Pedro Ribas 
dicha imposibilidad de fundamentar todo el conocimiento humano es lo que lleva a Kant a una experiencia que Hegel llamó experiencia de la conciencia.

\section{El primer contacto con la perplejidad}

En los Sueños de un visionario se establece una distinción entre conocer y entender donde se cifra el cambio de rumbo que toma Kant. Para Kant, conocer sólo otorga la facticidad, mientras que el entender da lugar a la compresión de la posibilidad de algo. Así, se distinguen también dos tipos de experiencias: las simples y las complejas. Las primeras son inaccesibles para el entender, por tanto, comprobaciones de hechos. Las segunda, por otro lado, son analizables y permiten la aplicación del método newtoniano. La impenetrabilidad es una experiencia simple, no analizable; hay, pues, desconocimiento de su posibilidad. Asimismo, también el espíritu en cuanto que lo opuesto a la impenetrabilidad de la materia también resulta ser inaccesible al entender. No hay, pues, conocimiento posible acerca del espíritu al que pudiera serle aplicado el método newtoniano. Aquí nos encontramos con una antinomia: la impenetrabilidad que podría plantearse del siguiente modo: parece evidente, a todas luces, que la materia es impenetrable. Sin embargo, está compuesta de átomos y si éstos se pueden separar, entonces, la materia seria penetrable, por tanto, la materia es penetrable e impenetrable al mismo tiempo. He aquí la perplejidad. Respecto a algo que conocemos no cabe ningún entendimiento en cuanto que sólo nos es dada la comprobación fáctica.

Además, aparece ya aquí aquel que despertó a Kant del sueño dogmático, Hume. "El nexo entre la causa y el efecto, considerado en abstracto, puede ser afirmado y negado sin incurrir en contradicción" 8 . La unión de causa y efecto no es a priori, sino sólo es dada por la experiencia de la causalidad. Por tanto, podríamos decir ya que "las síntesis filosóficas han de obtenerse después de los análisis y a partir de ellos, y por ello no cabe conocer la posibilidad de una cosa (síntesis a priori) ni el modo de influencia que ésta ejerce sobre otra (síntesis a posteriori)"

Entramos, pues, sin remedio, en la "beschwerlischste Verlegenheit", o sea, la completa ignorancia acerca de cualquier tema del espíritu y todo lo que se diga quedará reducido a mera opinión. Sin embargo, en cuanto que la razón se hace consciente del precipicio en donde se halla la perplejidad deja de tener efecto. La perplejidad no es una contradicción, sino la situación en la que cae la razón sin su culpa debido a preguntas cuya respuesta está

${ }^{8}$ FALGUERAS, Ignacio, "Perplejidad y filosofia trascendental en Kant". Pamplona, Universidad de Navarra, 1999, pág. 38.

9 Íbidem. Pág. 39. 
allende las capacidades del entendimiento humano. Las cuestiones que hacen que Kant caiga en la perplejidad no son otras que las antinomias y poseen las siguientes características:

a) Son proposiciones sobre objetos mundanos que pretenden referirse a los fundamentos mismos de todos los objetos.

b) Cada proposición tiene a su contraria como posible y no sólo en consonancia con los datos de la experiencia sino que una se puede deducir de la otra. Ambas proposiciones se encuentran perfectamente fundadas y con la misma solidez lógicas. De ahí su nombre.

c) Lo más característicos de ellas es su inesquivabilidad. La razón por sí sola tiende a plantearse tales problemas. Las ideas de yo y Dios no presuponen su objeto como dado, pero las ideas cosmológicas sí y es la razón la que tiende a alcanzar aquello que es incondicionado y absoluto.

Ante tal problema, Kant, como filósofo crítico que es, se encuentra sumido en una situación de inquietud que no es otra que la perplejidad, el no saber a qué atenerse a la hora de emitir algún juicio acerca de lo real. Sin embargo, la perplejidad es un problema que tiene su núcleo en la experiencia de la conciencia, es un problema gnoseológico que consiste en tener conceptos con los que no se puede pensar nada. Así, lo que toca ahora es la crítica, la imposición de límites al entendimiento para poder evitar la perplejidad.

\section{Conclusión. La prosecución de la perplejidad en la Crítica de la razón pura}

Así, hemos visto en qué consiste el problema de la perplejidad y como afecta al conocimiento del hombre y de qué manera le acontece. Sin embargo, resta señalar como Kant consigue avanzar en su filosofía al enfrentarse a dicho problema. La solución no será una solución, por así decirlo, total, o sea, de manera que el problema de la perplejidad desaparezca. La solución esla imposición de límites a la razón, esto es el ideal regulativo de la razón. Por tanto, más que superar el problema, la labor de Kant es bordearlo. La aceptación de la incapacidad de la razón para aceptar que no se puede decir, por ejemplo, del mundo, como totalidad de los fenómenos, que sea infinito y que no lo sea con la misma solidez y fundamento lógico lleva a Kant a mirar hacia el propio sujeto.

Dicho esto, la distinción entre fenómeno y noúmeno aparece de ésta manera en la obra crítica de Kant "Wenn die Welt ein an sich existierendes Ganzes ist: so ist sie entweder endlich, oder unendlich. Nun ist das erstere sowohl als das zweite falsch. Also ist es auch falsch, daß die Welt ein an sich existierendes Ganzes sei. Woraus denn folgt, daß Erscheinungen überhaupt außer unseren Vorstellungen nichts sind "10. El conocimiento,

${ }^{10}$ KANT, Immanuel. Kritik der reinen Vernunft II. Pág. 471. "Si el mundo es un todo existente en sí, entonces es, o bien finito, o bien infinito. Ahora bien, tanto lo primero como lo segundo es falso. Por 
por tanto, que se tiene del mundo no es más que en mis representaciones (Vorstellungen) que son sólo fenómenos. Ahora bien, ¿Cómo es posible que el hombre descubra unas leges entis si no tiene un contacto con lo nouménico? La solución de Kant se cifra en el salto a lo trascendental, o sea, a los esquemas trascendentales del sujeto dados en la estética trascendental que no son otros que el espacio y el tiempo, son, pues, leges mentis. ¿De dónde vienen las preguntas que la razón no puede rechazar y que tampoco puede responder? De las ideas de la razón cuyo uso ha de ser regulado por la razón pura.

La prosecución de las antinomias, pues, no es otra que el giro copernicano, o sea, la búsqueda de juicios sintéticos a priori, de los esquemas respecto a los cuales se rigen los fenómenos. Las respuestas, por tanto, a las preguntas que la razón humana se plantea y no puede rechazar no son posibles y lo único que cabe hacer respecto a las preguntas por Dios, el alma y el mundo es actuar como si (alsob) éstas existieran, pero no hay ninguna certeza en el plano teórico que nos muestre nada de lo nouménico. En conclusión, la perplejidad es la situación en la que cae la razón y que lleva a Kant a bordearla, a delimitar el alcance del conocimiento humano, a criticar a la razón bajo el tribunal de la razón pura.

\section{$* * * *$}

\section{Rafael Reyna Fortes}

rafaelreynafortes@gmail.com

consiguiente es igualmente falso que el mundo constituya un todo existente en sí mismo. De ello se sigue que los fenómenos no son nada fuera de nuestras representaciones". 